\title{
Was wissen wir über die regionale Variation der Gesundheitsausgaben?
} Und was bedeutet das für den Risikostrukturausgleich?

\section{DIRK GÖPFFARTH}

Dr. Dirk Göpffarth ist Referatsleiter im Bundesversicherungsamt in Bonn. Dieser Beitrag gibt die persönlichen Auffassungen des Verfassers wieder

\author{
Seit über 40 Jahre werden international regionale \\ Variationen untersucht. In Deutschland steht dieser \\ Forschungszweig noch am Anfang. Ein wichtiger \\ Zugang zur Untersuchung ist die Regionalisierung \\ des sektorübergreifenden Datensatzes aus \\ dem Risikostrukturausgleich. Der vorliegende \\ Beitrag zeigt, dass regionale Variationen in den \\ Ausgaben echt sind und nicht vollständig durch \\ Morbiditätsunterschiede erklärt werden können. \\ Es wird untersucht, ob siedlungsstrukturelle \\ Unterschiede oder Länderzugehörigkeit diese \\ Unterschiede erklären können und ob solche \\ Variablen zu einer höheren Zielgenauigkeit der \\ Krankenkassen-Zuweisungen führen könnten.
}

\section{Jahre Forschung zur regionalen Variation}

Die Erforschung regionaler Variation im Gesundheitswesen - sei es der Ausgaben, der Inanspruchnahme oder Behandlungsmuster - hat in den Vereinigten Staaten eine lange Tradition. Der Forschungszweig wurde vor ziemlich genau 40 Jahren durch einen bahnbrechenden Aufsatz von John Wennberg und Alan Gittelsohn begründet (Wennberg und Gittelsohn 1973, Wennberg 2010). In der Folge entstanden zahlreiche Veröffentlichungen zu dem Thema und der bekannte Dartmouth Atlas of Health Care. ${ }^{1}$ Die Untersuchungen bestätigten nicht nur die Existenz regionaler Variationen in den Gesundheitsausgaben, sondern auch, dass sich diese nur zu einem geringen Anteil erklären lassen.

Gemäß einer Studie des US Congressional Budget Office (CBO) lassen sich $30 \%$ der Variation der Ausgaben der staatlichen Rentnerversicherung Medicare durch Unterschiede in den Inputpreise und $20 \%$ durch Unterschiede in der Morbidität erklären (Congressional Budget Office 2008). Die für Vergütungsfragen von Medicare zuständige MedPAC-Kommission stellte fest, dass $40 \%$ der Variation durch Preise, Alter und Morbidität erklärt werden (Medpac 2003). Schließlich kommt eine Untersuchung von Bernstein et al. (2011) zu dem Ergebnis, dass $30 \%$ bis $45 \%$ der regionalen Variation erklärt werden kann. Damit bleibt aber weit über die Hälfte der Variation unerklärt.

Eine politische Dimension erhielt die Diskussion, als eine breit angelegte Untersuchung zu dem Ergebnis kam, dass höhere Gesundheitsausgaben mitnichten mit einer höheren Qualität der Versor-

1 Siehe http://www.dartmouthatlas.org. Dort findet sich auch eine umfangreiche Bibliographie. 
gung verbunden sind, sondern dass es vielmehr sogar Hinweise auf negative Korrelationen gibt (Fisher et al. 2003a, Fisher et al. 2003b). Die Schlussfolgerung liegt nahe, die Variationen als Zeichen für Ineffizienzen zu werten, die, wenn sie gehoben werden könnten, zu Einsparpotenzialen bei gleicher vermuteter Qualität führten. Sutherland et al. (2009) gehen von einem Einsparpotenzial von $30 \%$ aus. Diese Sichtweise hat auch Eingang in Präsident Obamas Gesundheitsreform in Form von Vergütungsabschlägen in Hochkostenregionen gefunden.

In den letzten Jahren kam es zu einer zunehmenden kritischen Auseinandersetzung mit dieser Sichtweise. Kritiker weisen auf den fehlenden Nachweis eines kausalen Zusammenhangs zwischen der Variation und Ineffizienzen hin (Sheiner 2013) und vermuten die Ursachen für die nicht erklärte Varianz in Morbiditätsunterschieden, die nicht durch Leistungsdaten nachgewiesen werden können (Bernstein et al. 2011, Doyle 2011, Reschovsky et al. 2011, Zuckerman et al. 2010) oder in Kodiervariationen (Song et al. 2010).

Zur Klärung dieser Kontroversen wurde das Institute of Medicine beauftragt, ein umfassendes Gutachten zu diesem Thema vorzulegen (Newhouse et al. 2013). Das Gutachten bestätigt das Ausmaß nicht erklärbarer Variation und die Tatsache, dass diese Variation nicht mit Unterschieden in der Qualität der Versorgung korreliert ist.

\section{Diskussion in Deutschland steht erst am Anfang}

Der Dartmouth Atlas und die Herangehensweise an die Untersuchung regionaler Variationen haben in vielen Ländern Nachahmer gefunden. ${ }^{2}$ In Deutschland - einem Land mit hohen Gesundheitsausgaben aber eher durchschnittlichen Ergebnisindikatoren - lassen sich auch größere Ineffizienzen vermuten. Trotzdem existieren hier erst wenige Studien, vermutlich aus Gründen einer fehlenden Verfügbarkeit regionaler Daten.

In den letzten Jahren haben sich sektoral begrenzte Darstellungen etabliert. Der Versorgungsatlas des Zentralinstituts für die kassenärztliche Versorgung ${ }^{3}$ mit den Daten der vertragsärztlichen Versorgung und der Faktencheck Gesundheit der Bertelsmann-Stiftung ${ }^{4}$ mit den Daten der stationären Versorgung orientieren sich mit ihren interaktiven Karten erkennbar am Dartmouth Atlas. Auch andere Studien untersuchen regionale Variationen im Rahmen der vertragsärztlichen oder stationären Versorgung. ${ }^{5}$

Untersuchungen zur sektorübergreifenden regionalen Variation in den Gesundheitsausgaben basieren hauptsächlich auf den Daten des Risikostrukturausgleichs. Der Risikostrukturausgleich ist das Finanzausgleichsverfahren zwischen den gesetzlichen Krankenkassen. Seit dem 1.1.2009 wird er morbiditätsorientiert durchgeführt; zu diesem Zweck werden für alle GKV-Versicherten von den Krankenkassen die Diagnosen aus der vertragsärztlichen und stationären Versorgung sowie die Arzneimittelverordnungen versichertenbezogen gemeldet. Die Jahresausgaben kommen für eine Stichprobe von knapp $7 \%$ der GKV-Versicherten hinzu; seit 2011 werden diese Angaben für alle Versicherten gemeldet. Zur Begrenzung der regionalen Verteilungswirkung durch die Einführung des Gesundheitsfonds fand in den Jahren 2009 und 2010 die sog. „Konvergenzklausel“ Anwendung ( $\sqrt{ } 272$ SGB V). Zu diesem Zweck wurden in diesen Jahren die Datenmeldungen um ein Regionalmerkmal ergänzt (5-stelliger Kreisgemeindeschlüssel).

Neben der Frage der länderbezogenen Umverteilungen wird im Risikostrukturausgleich auch die Notwendigkeit einer Regionaldimension zur Verbesserung der Zielgenauigkeit der Zuweisungen diskutiert. Nicht zuletzt durch die Schließung der in den Hochkostenregionen Hamburg und Berlin konzentrierten City-BKK wegen mangelnder wirtschaftlicher Leistungsfähigkeit wurde die Frage aufgeworfen, ob regionale Ausgabenunterschiede im Risikostrukturausgleich zu berücksichtigen seien. Vor diesem Hintergrund untersuchte der Wissenschaftliche Beirat zur Weiterentwicklung des Risikostrukturausgleichs beim Bundesversicherungsamt diese Frage (Drösler et al. 2011). Hierzu stellte er regionale Morbiditäts- und Ausgabenvariationen dar und untersuchte die im Risikostrukturausgleich entstehenden regionalen Über- und Unterdeckungen auf Kreisebene. Dabei wies der Beirat insbesondere auf Unterdeckungen in den Kernstädten und Überdeckungen im umliegenden Verdichtungsraum hin. Eine Einbeziehung von Regionalmerkmalen auf Länderebene lehnt der Beirat hingegen ab. Weitere Analysen auf diesem Datensatz präsentiert Göpffarth (2011).

\section{Datengrundlage}

Die bisherigen Analysen auf der Grundlage der Daten des Risikostrukturausgleichs basieren hinsichtlich der regionalen Ausgaben auf eine Auswertung der 7\%-Stichprobe. Aufgrund der schiefen Verteilung der Gesundheitsausgaben ist es aber nicht auszuschließen, dass diese Ergebnisse durch Stichprobenfehler verzerrt sind. Dafür gibt es tatsächlich Hinweise: Im Jahr 2009 wies Bad Kissingen mit 2.735 Euro die höchsten Ausgaben auf. Selbst nach Standardisierung lagen die Ausgaben noch bei 2.666 Euro. Im Folgejahr 2010 waren die Ausgaben in Bad Kissingen mit 2.489 Euro nach wie vor hoch, aber nicht mehr so auffällig. Im Jahr 2010 hingegen wurden die höchsten Ausgaben mit 2.834 Euro in Pfaffenhofen/Ilm verzeichnet; nach Standardisierung betrugen diese sogar 3.144 Euro. Im Vorjahr 2009 lagen die Ausgaben in Pfaffenhofen/Ilm noch bei unterdurchschnittlichen 1.951 Euro. Diese Schwankungen sind wohl kaum real; umso wichtiger wäre daher eine Kontrolle der Ergebnisse auf der Grundlage einer Vollerhebung.

Obwohl das Regionalmerkmal nur in den Jahren 2009 und 2010 erhoben wurde, und die Vollerhebung der Ausgaben im Risikostrukturausgleich erst 2011 begann, ist eine Verknüpfung möglich. Dies liegt daran, dass der Risikostrukturausgleich als prospektives System angelegt ist: Ausschlaggebend für die Zuweisungen sind die Diagnosen des Vorjahres; die Höhe der Zuweisungen wiederum orientiert sich an den Folgekosten im Folgejahr. Um dies berechnen zu können, müssen die Daten jeweils zweier Jahre miteinander verknüpfbar sein. Somit lassen sich auch die vollerhobenen Ausgaben des Jahres 2011 mit dem Regionalmerkmal des Jahres 2010 verknüpfen. Offensichtlich funktioniert diese Verknüpfung nicht für Versicherte des Jahres 2011, die im Jahr 2010 noch nicht versichert waren - d.h. Neugeborene, Zuwanderer, Rückkehrer aus PKV

2 Z.B in Großbritannien: National Health Service 2010 . Einen Überblick über Studien aus verschiedenen Ländern vermittelt die Seite http://wennbergcollaborative.org.

3 http://www.versorgungsatlas.de

4 http://www.faktencheck-gesundheit.de, vgl. Bertelsmann Stiftung 2011.

5 Vgl. Greube et al. 2011, die Beiträge im Krankenhausreport 2012 (Augurzky und Klauber 2012) sowie Augurzky et al. 2013. 
etc. Allerdings lassen sich so für $97,7 \%$ der Versicherte des Jahres 2011 Angaben zum Wohnsitz machen.

\section{Regionale Variationen auf Kreisebene}

Angaben zu regionalen Unterschieden in der Morbidität lagen bereits bisher auf der Grundlage einer Vollerhebung vor (Drösler et al. 2011, Göpffarth 2011). Mit Morbidität wird hier die im Rahmen des Risikostrukturausgleichs über die Angaben Alter, Geschlecht, Bezug einer Erwerbsminderungsrente sowie über die Diagnosen und Verordnungen für 80 schwerwiegende oder kostenintensiv-chronische Erkrankungen erfasste Morbidität gemeint (RSA-Risikofaktor). Diese Angaben weisen eine hohe Kons$\operatorname{tanz}$ auf. So liegt der Korrelationskoeffizient der RSA-Risikofaktoren je Kreis zwischen den Jahren bei $r=0,9946(2009$ zu 2010) bzw. $r=0,9930$ (2010 zu 2011).

Bei den regionalen Ausgaben konnte eine solche Stabilität nicht festgestellt werden - die Ausgaben wurden aber auch bislang im Rahmen einer Stichprobe erfasst. Die Korrelation der Ausgaben zwischen den Jahren 2009 und 2010 lag bei $r=0,8682$. Die Korrelation zwischen den Stichprobenergebnissen aus 2010 und den vollerhobenen Ausgaben aus 2011 lag in derselben Größenordnung $(r=0,8485)$. Ob sich von der Vollerhebung eines Jahres zur Vollerhebung eines anderen Jahres eine größere Stabilität ergibt, kann nicht gesagt werden, da die nächste Vollerhebung des Jahres 2012 nicht mehr regionalisiert werden kann. Die Spanne der Pro-Kopf-Ausgaben auf Kreisebene hat sich in der Vollerhebung 2011 (1.876 Euro bis 2.727 Euro) gegenüber der Stichprobe 2010 (1.805 Euro bis 2.834 Euro) nur leicht reduziert. Der Variationskoeffizient hat sich geringfügig von 8,12 auf 7,83 reduziert.

Entscheidend wird es, wenn man die beiden Seiten - Morbidität und Ausgaben - zusammenbringt. Dies erfolgt im Rahmen der standardisierten Leistungsausgaben. Die Standardisierung der Leistungsausgaben erfolgt anhand der Risikomerkmale des Risikostrukturausgleiches. Die Stabilität der Höhe der standardisierten Leistungsausgaben je Kreis zwischen den Jahren war bislang nicht besonders hoch (zwischen 2009 und $2010 \mathrm{r}=0,612$ und zwischen 2010 und $2011 \mathrm{r}=0,5235$ ).

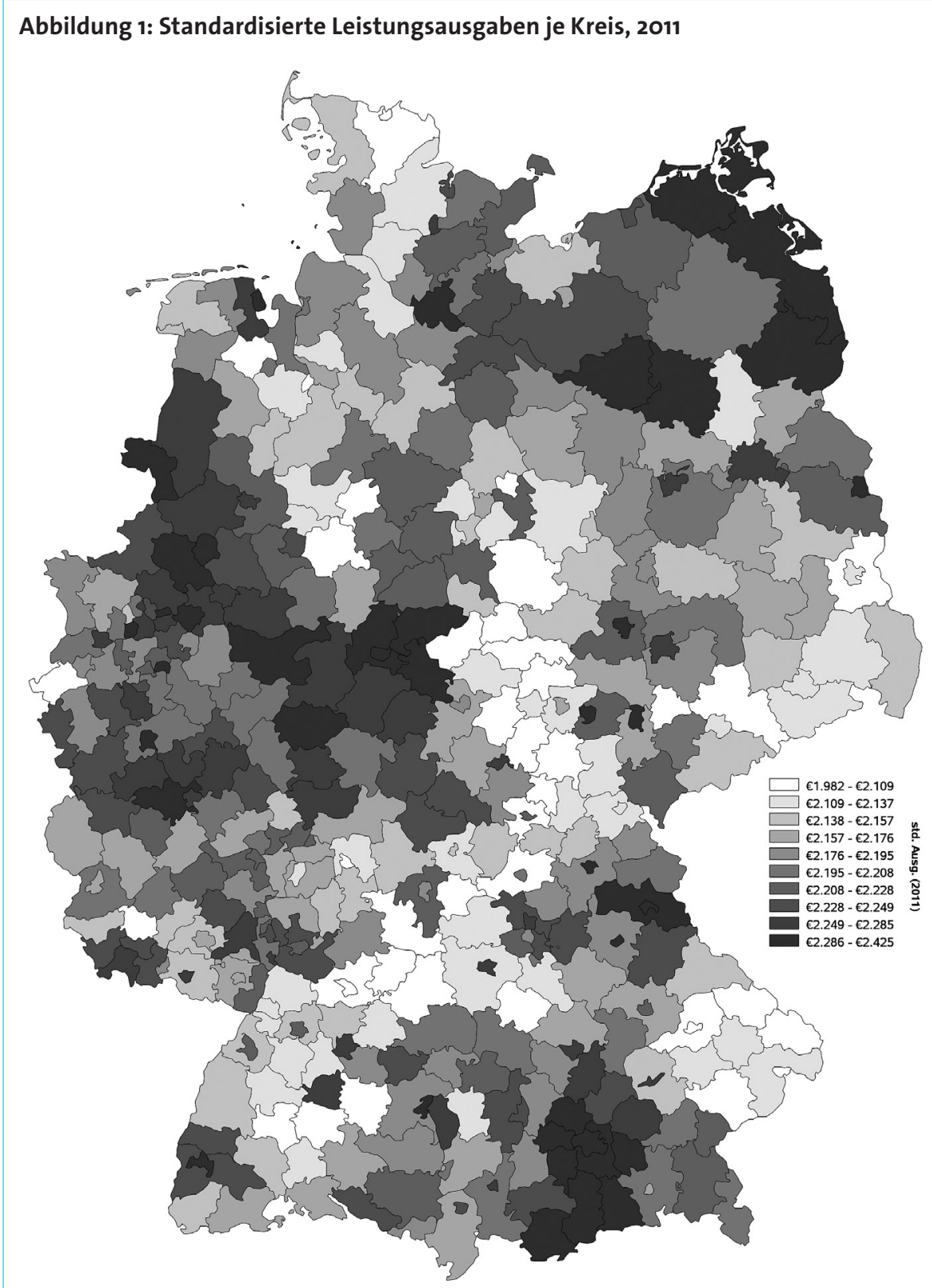

Quelle: Eigene Darstellung

Die Unterschiede zwischen den standardisierten Leistungsausgaben je Kreis, dargestellt in Abbildung 1, haben zwei Interpretationen: Zum einen zeigen die Unterschiede die Varianz in den Ausgaben zwischen den einzelnen Kreise an, die nicht durch demographische Unterschiede und Unterschiede in der dokumentierten Morbidität erklärt werden können. Hier haben sich durch die Umstellung auf die Vollerhebung sowohl Spanne als auch Varianz erheblich reduziert. Im Jahr 2009 lag die Spanne bei 1.852 Euro bis 2.666 Euro und der Variationskoeffizient bei 4,72. Die Spanne im Jahr 2010 ging von 1.908 Euro bis 3.144 Euro bei einem Variationskoeffizienten von 5,01. Mit dem Umstieg auf die Vollerhebung reduziert sich die Spanne auf 1.982 Euro bis 2.425 Euro und der Variationskoeffizient auf 3,27. Die regionale Verteilung der Über- und Unterdeckungen ist sehr ähnlich zu den Vorjahren. Offensichtlich hat die Stichprobe zu einzelnen Ausreißern geführt, die Spanne und Varianzen erhöhen, aber nicht zu einem systematisch anderen Bild führen. Allerdings ist nun der Anteil der Variation in den Ausgaben je Kreis, die durch die Faktoren des Risikostrukturausgleichs erklärt werden können, von 44 \% (Göpffarth 2011) auf nunmehr knapp 60 \% angestiegen. Diesen Effekt der zunehmenden Varianzerklärung kann man auch den Boxplots in Abbildung 2 entnehmen. 


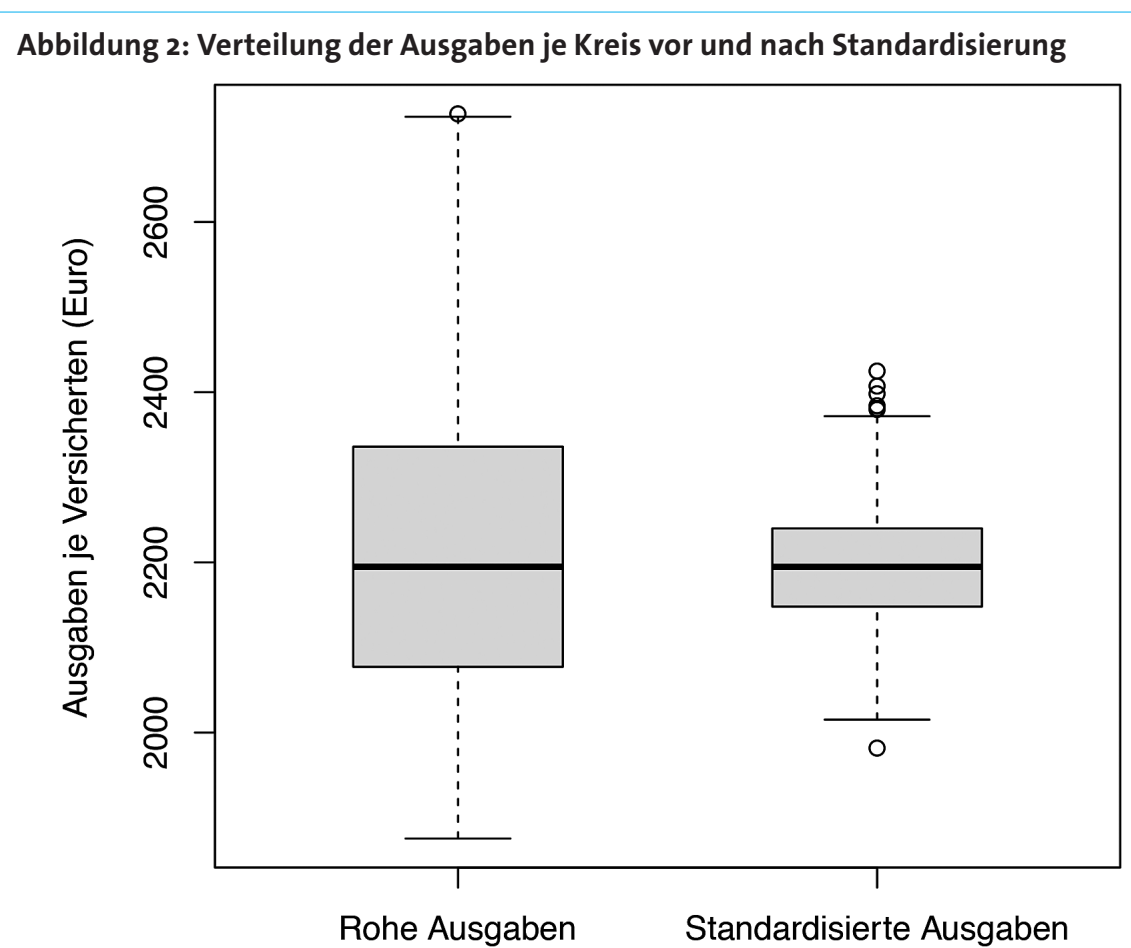

Quelle: Eigene Darstellung
Die 60\%ige Reduzierung der Varianz kann als Bestätigung des Diktums von Klaus Jacobs gelten: Der „Morbi-RSA regionalisiert automatisch [...] Ist die Krankheitslast in einer Region besonders hoch, erhalten die Krankenkassen für mehr Geld“" (Jacobs 2010).

Die zweite Interpretation der Abbildung 1 liegt in dem Bezug zum Risikostrukturausgleich. Da zur Standardisierung genau die Risikofaktoren des Risikostrukturausgleichs herangezogen wurden, erhält die standardisierte Bevölkerung gerade die bundesdurchschnittliche Zuweisung in Höhe von 2.207 Euro. Liegen die standardisierten Leistungsausgaben z.B in Weiden in der Oberpfalz bei 2.425 Euro, so haben die dort tätigen Krankenkassen im Durchschnitt Unterdeckungen von über 200 Euro je Versicherten. Bei standardisierten Leistungsausgaben in Höhe von 1.982 Euro in Flensburg entstehen hingegen durchschnittliche Überdeckungen von über 200 Euro. Solche Über- und Unterdeckungen bergen die Gefahr einer regionalen Risikoselektion (Bauhoff 2012), so dass sich die Frage nach einer Regionalkomponente im Risikostrukturausgleich stellt.

Bemerkenswert ist, dass die Variation der standardisierten Gesamtausgaben mit einem Variationskoeffizient von 3,27 die dortigen Versicherten entsprechend geringer liegt als die Variation der standardisierten Ausgaben der einzelnen Leistungsbereiche (Ärzte: 8,21, Arzneimittel: 6,33, Krankenhaus: 6,38, Sonstige: $8,05)$. Offensichtlich bestehen zwischen den einzelnen Leistungsbereichen (z.B. zwischen Ärzten und Krankenhaus oder zwischen Ärzten und Arzneimittel) Kompensationseffekte. Dies spricht auch für die Notwendigkeit einer sektorübergreifenden Betrachtung und zeigt die Grenzen sektorspezifischer Betrachtungen auf.

\section{Erklärung der verbliebenen Variation}

Bei der Suche nach Erklärungsansätzen für die nach der Standardisierung durch den morbiditätsorientierten Risikostrukturausgleich verbliebenen Variationen und möglichen Schlussfolgerungen für die Ausgestaltung des Risikostrukturausgleichs gibt es im Wesentlichen zwei
Ansätze. Während Gaßner et al. (2011) für eine Regionalvariable auf Länderebene plädieren, führen Drösler et al. (2011) nalfaktoren im Risikostrukturausgleich, die auf Ländergrenzen abstellt, aus wissenschaftlicher Sicht zu kurz greifen würde.“ Stattdessen stellen sie Überlegungen an, die in die Richtung einer Berücksichtigung siedlungsstruktureller Elemente gehen, werfen aber auch die Frage auf, ob diese Problematik im Rahmen des Risikostrukturausgleichs zu lösen sei. Beide Betrachtungsperspektiven sollen

Im Rahmen der Laufenden Raumbeobachtung unterscheidet das Bundesinstitut für Bau-, Stadt- und Raumforschung (BBSR) vier siedlungsstrukturelle Kreistypen: Kreisfreie Großstädte, städtische Kreise, ländliche Kreise mit Verdichtungsansätzen sowie dünn besiedelte ländliche Kreise. Hierfür wird der Bevölkerungsanteil in Groß- und Mittelstädten sowie die Einwohnerdichte der Kreisregion herangezogen. Tabelle 1 zeigt die durchschnittlichen Über- bzw. Unterdeckungen im Risikostrukturausgleich nach siedlungsstrukturellem Kreistyp auf. Wie bereits von Drösler et al. (2011) - anhand einer älteren Typologie der Kreise - aufgezeigt, weisen insbesondere der stark verstädterte Raum Unterdeckungen auf, während im ländlichen Raum durchschnittlich Überdeckungen erreicht werden. Für Versicherte in kreisfreien Großstädten erzielen Krankenkassen im Durchschnitt 39 Euro weniger an Zuweisungen als sie an Ausgaben realisieren. Die Unterdeckung entsteht in erster Linie im Bereich der vertragsärztlichen Versorgung. Dieser Sektor trägt umgekehrt auch maßgeblich zu den Überdeckungen auf dem Lande bei.

Etwas deutlicher wird der Zusammenhang, wenn man bei der Aggregation der Kreise nicht auf den siedlungsstrukturellen Typ, sondern auf räumliche Lage - d.h. peripher oder zentral - abstellt. Die aus, dass „eine Einbeziehung von Regioim Folgenden dargestellt werden.

Tabelle 1: Über- und Unterdeckung im RSA nach siedlungsstrukturellem Kreistyp, 2011

\begin{tabular}{l|r|r|c|c|}
\hline & \multicolumn{1}{|c|}{ Alle } & Ärzte & Arzneimittel & Krankenhaus \\
\hline Kreisfreie Großstädte & $-39,2 €$ & $-28,5 €$ & $5,8 €$ & $-7,6 €$ \\
\hline Städtische Kreise & $4,0 €$ & $0,4 €$ & $-3,0 €$ & $3,7 €$ \\
\hline Ländliche Kreise mit & $34,9 €$ & $18,5 €$ & $-1,0 €$ & $10,0 €$ \\
\hline Verdichtungsansatz & $21,2 €$ & $30,0 €$ & $-1,5 €$ & $-7,3 €$
\end{tabular}

Quelle: Eigene Darstellung 
Tabelle 2: Über- und Unterdeckung im RSA nach siedlungsstrukturellem Kreistyp, 2011

\begin{tabular}{|l|c|c|c|c|}
\hline & Alle & Ärzte & Arzneimittel & Krankenhaus \\
\hline Sehr zentral & $-25,1 €$ & $-17,8 €$ & $3,3 €$ & $-7,4 €$ \\
\hline Zentral & $12,0 €$ & $3,0 €$ & $-3,1 €$ & $9,9 €$ \\
\hline Peripher & $37,9 €$ & $26,5 €$ & $-0,6 €$ & $7,1 €$ \\
\hline Sehr peripher & $-9,7 €$ & $54,2 €$ & $-16,5 €$ & $-38,5 €$ \\
\hline
\end{tabular}

Quelle: Eigene Darstellung

räumliche Lage wird vom BBSR anhand der erreichbaren Tagesbevölkerung ermittelt. Die Über- und Unterdeckungen nach räumlicher Lage finden sich in $\mathrm{Ta}$ belle 2. Hier sieht man die maßgeblich durch die vertragsärztliche Versorgung getriebenen Unterdeckungen in sehr zentralen Lagen, während in peripheren Lagen erheblich Überdeckungen aus der vertragsärztlichen zu verzeichnen sind. In sehr peripheren Gebieten werden diese Überdeckungen aber durch Mehrausgaben in der Arzneimittel- und Krankenhausversorgung kompensiert.
Auf Landesebene schwanken die Deckungsbeträge zwischen einer durchschnittlichen Unterdeckung von 156 Euro in Hamburg bis zu einer durchschnittlichen Überdeckung von 79 Euro in Thüringen. Dass aber die Schwankungen innerhalb der Bundesländer sehr groß sind, und in jedem Flächenland sowohl über- wie überdeckte Kreise existieren, kann Abbildung 1 entnommen werden.

Welchen Anteil an der nach Standardisierung verbliebenen Varianz können die Kreistypen bzw. die Landeszugehö- rigkeit erklären? Hierzu wurde eine populationsgewichtete lineare Regression mit den Deckungsbeträge je Versicherten je Kreis als abhängige Variable und den Kreistypen bzw. die Länderzugehörigkeit als unabhängige Variablen durchgeführt. Die Ergebnisse sind in Tabelle 3 dokumentiert. Es zeigt sich, dass die Kreistypen etwa $12 \%$ bis $14 \%$, die Länderzugehörigkeiten hingegen knapp $27 \%$ der verbleibenden Variation erklären können ( $\mathrm{R}^{2}$-Wert). Kreistyp und Länderzugehörigkeit zusammen erklären etwa ein Drittel der verbliebenen Variation.

\section{Einbindung in den Risikostrukturausgleich}

Es soll zunächst empirisch überprüft werden, welche Effekte eine Einbindung eines Regionalmerkmals - entweder in Form einer Variable für den Kreistyp oder in Form einer Variable für die Länderzugehörigkeit des Versicherten - in

Tabelle 3: Regression der Deckungsbeträge auf Kreistypen und Länder, 2011

\begin{tabular}{|c|c|c|c|c|c|c|c|c|}
\hline & \multirow{2}{*}{\multicolumn{2}{|c|}{ Modell 1}} & \multirow{2}{*}{\multicolumn{2}{|c|}{ Modell 2}} & \multirow{2}{*}{\multicolumn{2}{|c|}{ Modell 3}} & \multirow{2}{*}{\multicolumn{2}{|c|}{ Modell 4}} \\
\hline & & & & & & & & \\
\hline & B & $\begin{array}{l}\text { Standard- } \\
\text { fehler }\end{array}$ & B & $\begin{array}{l}\text { Standard- } \\
\text { fehler }\end{array}$ & B & $\begin{array}{l}\text { Standard- } \\
\text { fehler }\end{array}$ & B & $\begin{array}{l}\text { Standard- } \\
\text { fehler }\end{array}$ \\
\hline (Konstante) & 79,468 & 18,493 & $-25,140$ & 5,002 & $-39,186$ & 6,372 & 35,553 & 19,392 \\
\hline Schleswig-Holstein & $-50,090$ & 25,090 & & & & & $-48,064$ & 24,179 \\
\hline Hamburg & $-235,403$ & 28,622 & & & & & $-191,489$ & 28,597 \\
\hline Niedersachsen & $-66,117$ & 21,058 & & & & & $-63,419$ & 20,321 \\
\hline Bremen & $-38,044$ & 39,234 & & & & & 5,870 & 38,527 \\
\hline Nordrhein-Westfalen & $-99,021$ & 19,663 & & & & & $-74,808$ & 19,769 \\
\hline Hessen & $-92,502$ & 21,822 & & & & & $-77,826$ & 21,476 \\
\hline Rheinland-Pfalz & $-88,454$ & 23,422 & & & & & $-79,155$ & 22,848 \\
\hline Baden-Württemberg & $-47,502$ & 20,483 & & & & & $-32,951$ & 20,396 \\
\hline Bayern & $-93,202$ & 20,214 & & & & & $-87,351$ & 19,514 \\
\hline Saarland & $-91,515$ & 33,671 & & & & & $-79,229$ & 33,110 \\
\hline Berlin & $-128,944$ & 24,225 & & & & & $-85,029$ & 24,550 \\
\hline Brandenburg & $-60,657$ & 25,540 & & & & & $-64,864$ & 24,811 \\
\hline Mecklenburg-Vorpommern & $-122,247$ & 28,515 & & & & & $-126,274$ & 27,658 \\
\hline Sachsen & $-22,846$ & 22,869 & & & & & $-13,584$ & 22,182 \\
\hline Sachsen-Anhalt & $-36,103$ & 25,829 & & & & & $-33,712$ & 24,873 \\
\hline Lage: zentral & & & 37,135 & 7,975 & & & & \\
\hline Lage: peripher & & & 63,078 & 8,825 & & & & \\
\hline Lage: Sehr peripher & & & 15,471 & 20,832 & & & & \\
\hline Städt. Kreise & & & & & 43,140 & 8,316 & 31,629 & 8,423 \\
\hline Ländl. Kreis mit Verdichtung & & & & & 74,058 & 10,230 & 56,533 & 10,488 \\
\hline Dünn besiedl. Ländl. Kreise & & & & & 60,399 & 10,757 & 52,780 & 11,743 \\
\hline R-Quadrat & & 0,2669 & & 0,1242 & & 0,1373 & & 0,3267 \\
\hline
\end{tabular}

Quelle: Eigene Darstellung 
den Risikostrukturausgleich hätte. Dies geschieht losgelöst von der normativen Fragestellung, ob eine solche Einbindung auch erfolgen sollte. Technisch werden in den Modell jeweils Dummyvariablen je Versicherten für die 16 Bundesländer bzw. den vier Kreistypen gebildet und in die Regression - neben AGG, EMG und HMG - einbezogen.

Zunächst sollte durch eine Einbindung eines Regionalmerkmales dreierlei erreicht werden. Zum einen sollten die in Abbildung 1 aufgezeigten Über- und Unterdeckungen reduziert werden, da von ihnen eine Gefahr regionaler Risikoselektionsstrategien ausgehen könnte. Zum anderen existieren auch zwischen den Krankenkassen selber erhebliche Unterschiede in den Deckungsquoten, die aus Unterschieden in der Wirtschaftlichkeit allen nicht erklärt werden können. So lag die Spanne der Deckungsquoten im Jahr 2011 zwischen 86,6 \% und 113,3\% ${ }^{6}$ Die mittlere absolute prozentuale Abweichung (MAPE) der Zuweisung von den Ausgaben lag bei 2,65 \%. Möglicherweise sind im Risikostrukturausgleich nicht kompensierte regionale Ausgabenunterschiede eine Ursache hierfür. Schließlich misst sich die Zielgenauigkeit auf Individualebene durch das statistischen Bestimmtheitsmaß R ${ }^{2}$, das im Referenzmodell bei 23,8290\% lag.

Beim Kreistypmodell wird nur auf den siedlungsstrukturellen Kreistyp abgestellt, da dieser in der vorangegangenen Analyse (Tabelle 3) die höhere Erklärungskraft aufwies. Dieses Modell mit einem $\mathrm{R}^{2}$-Wert von 23,8305\% würde die Unter- und Überdeckungen nach Kreistyp eliminieren. Trotzdem bleibt die Spanne der Über- und Unterdeckungen von jeweils knapp über 200 Euro auf Kreisebene unverändert. Während im Status quo ohne Regionalmerkmal 189 Kreise mit 30,1 Mio. GKV-Versicherten eine Über- oder Unterdeckung vom im Betrag mehr als 50 Euro aufwiesen, sind das nachher noch 178 Kreise mit 27,7 Mio. Versicherte. Die regionalen Überund Unterdeckungen auf Kreisebene werden also nur unwesentlich verringert. Auch auf Kassenebene reduziert sich die Spanne nur auf $87,1 \%$ bis $113,2 \%$ bei einem MAPE von 2,59\%.

Das Ländermodell führt auf Individualebene zu keiner besseren Zielgenauigkeit; der R ${ }^{2}$-Wert fällt auf 23,4607 \%. Dies gilt aber auch auf Kreisebene: Die Spanne der Deckungsbeträge steigt an; die maximale Überdeckung liegt nun bei 265 Euro. Mit absoluten Über- oder Unterdeckungen von mehr als 50 Euro bleiben 189 Kreise mit 30,0 Mio. Versicherte betroffen. Da in jedem Flächenland sowohl über- als auch unterdeckte Kreise existieren, würde dieses Modell in einem im Durchschnitt unterdeckten Land mehr Mittel zuweisen und damit zwar die Unterdeckungen in den einen Kreisen reduzieren, gleichzeitig aber auch die Überdeckungen in den anderen Kreisen erhöhen. Umgekehrt verhielt es sich in im Durchschnitt überdeckten Ländern. Auf Kassenebene schließt sich

\section{Eine Einbindung von Regionalvariablen verbessert die Zielgenauigkeit allenfalls marginal.}

die Spanne der Deckungsbeiträge im Ländermodell etwas stärker als im Kreistypmodell, bleibt aber immer noch bei $87,3 \%$ bis $112,0 \%$. Das MAPE liegt bei 2,45 .

Auch mit einer Kombination aus Ländermodell und Kreistypmodell gelingt es nicht, die Zielgenauigkeit der Zuweisungen auf Ebene der individuellen Versicherten, der Kreise und der Krankenkassen zu erhöhen.

\section{Diskussion}

Die hier durchgeführten Analysen haben folgendes gezeigt:

- Regionale Variationen in den Ausgaben sind echt, und kein statistisches Artefakt der bisherigen Stichprobenerhebungen.

- Zum überwiegenden Teil können diese Variationen durch die demographischen und morbiditätsorientierten Risikomerkmale des Risikostrukturausgleichs erklärt werden. Trotzdem verbleiben nicht unerhebliche Variationen auf Kreisebene.

- Es besteht die Gefahr, dass diese Variationen regional tätige Krankenkassen benachteiligen bzw. Ausgangspunkt von regionalen Risikoselektionsstrategien sein könnten.

- Landeszugehörigkeit und siedlungsstruktureller Kreistyp können etwa ein Drittel dieser verbliebenen Variationen erklären.
- Eine Einbindung dieser Variablen in den Risikostrukturausgleich führt aber allenfalls zu einer minimalen Verbesserung der Zielgenauigkeit des Risikostrukturausgleichs. Sowohl die Über- und Unterdeckungen auf Kreisebene, als auch die Spanne der Deckungsquoten bleibt fast unverändert bestehen.

Gegen die Einbindung eines Regionalmerkmals in den Risikostrukturausgleich werden auch normative Argumente vorgebracht. Zum einen muss gefragt werden, inwieweit die regionalen Ausgabenunterschiede aus Sicht der Krankenkassen exogen sind, oder ob sie nicht doch teilweise auch durch die Aktivitäten der Krankenkassen beeinflusst werden. Doch selbst wenn die Faktoren exogen sind, stellt sich die Frage ob der Ausgleich in den Risikostrukturausgleich gehört oder ob hier nicht eher regionale Differenzierungen bei der Beitragserhebung oder in den Vergütungssystemen gefragt sind. Diese Fragen können aber insofern zurückgestellt werden, als dass die diskutierten Modelle schon rein empirisch nicht überzeugen können.

Um überzeugende Modelle zu entwickeln bedarf es daher eines größeren Verständnisses, welche Faktoren die regionalen Ausgabenunterschiede determinieren. Es ist daher zu begrüßen, dass im Koalitionsvertrag der Großen Koalitionsvertrag vorgesehen ist, das Regionalmerkmal wieder zu erheben. Die hier präsentierten Analysen haben nämlich auch gezeigt, dass der Aussagefähigkeit sektoraler Analysen enge Grenzen gesetzt sind, und dass für die Analyse regionaler Variationen tatsächlich ein sektorübergreifender Datensatz wie der des Risikostrukturausgleichs notwendig ist.

6 Um sicherzustellen, dass die hier ausgewiesenen Effekte nicht aus methodischen Problemen aufgrund einer fehlenden Annualisierung der Ausgaben Verstorbener resultiert, wurden die Ausgaben hier zu analytischen Zwecken - anders als bei der Durchführung des Verfahrens - annualisiert. Die Spanne weicht daher von der tatsächlichen des Jahres 2011 (88,0 \% bis $114,5 \%$ ) etwas ab. 


\section{Literatur}

Augurzky, Boris; Klauber, Jürgen (2012): Schwerpunkt: Regionalität. Mit 90 Tabellen; [mit Online-Zugang]. Stuttgart: Schattauer (Krankenhaus-Report, 2012).

Augurzky, Boris; Kopetsch, Thomas; Schmitz, Hendrik (2013): What accounts for the regional differences in the utilisation of hospitals in Germany? In: Eur J Health Econ 14 (4), S. 615-627.

Bauhoff, Sebastian (2012): Do health plans risk-select? An audit study on Germany's Social Health Insurance. In: Journal of Public Economics 96 (9-10), S. 750-759.

Bernstein, Jill; Reschovsky, James D.; White, Chapin (2011): Geographic Variation in Health Care: Changing Policy Directions. National Institute for Health Care Reform. Washington DC (Policy Analysis, 4).

Bertelsmann Stiftung (2011): Faktencheck Gesundheit. Regionale Unterschiede in der Gesundheitsversorgung. Bertelsmann Stiftung. Gütersloh.

Congressional Budget Office (2008): Geographic Variation in Health Care Spending. Congressional Budget Office. Washington.

Doyle, Joseph J. (2011): Returns to Local-Area Health Care Spending: Evidence from Health Shocks to Patients Far From Home. In:

American Economic Journal: Applied Economics 3 (3), S. 221-243.

Drösler, Saskia; Hasford, Joerg; Kurth, Bärbel-Maria; Schaefer, Marion; Wasem, Jürgen (2011): Evaluationsbericht zum Jahresausgleich 2009 im Risikostrukturausgleich. Wissenschaftlicher Beirat zur Weiterentwicklung des Risikostrukturausgleichs. Bonn.

Fisher, Elliott S.; Wennberg, David E.; Stukel, Thérèse A.; Gottlieb, Daniel J.; Lucas, F. L.; Pinder, Etoile L. (2003a): The implications of regional variations in Medicare spending. Part 1: the content, quality, and accessibility of care In: Ann. Intern. Med. 138 (4), S. 273-287.

Fisher, Elliott S.; Wennberg, David E.; Stukel, Thérèse A.; Gottlieb, Daniel J.; Lucas, F. L.; Pinder, Etoile L. (2003b): The implications of regional variations in Medicare spending. Part 2: health outcomes and satisfaction with care. In: Ann. Intern. Med. 138 (4), S. 288-298.

\section{Gaßner, Maximilian; Göpffarth, Dirk;}

Wittmann, Rüdiger (2011): Spielräume für eine Dezentralisierung im Rahmen des Systems Gesundheitsfonds. In: Eberhard Wille und Klaus Knabner (Hg.): Dezentralisierung und Flexibilisierung im Gesundheitswesen. 1. Aufl. Frankfurt, M, Berlin, Bern, Bruxelles, New York, NY, Oxford, Wien: Lang, S. 53-81.

Göpffarth, Dirk (2011): Regionalmerkmale im Risikostrukturausgleich. Ein Beitrag zum funktionalen Wettbewerb und zu bedarfsgerechter Versorgung? In: Uwe Repschläger (Hg.): BARMER Gesundheitswesen aktuell 2011. [Beiträge und Analysen]. Wuppertal: Barmer GEK, S. 16-40.

Greube, Thomas C.; Dörning, H.; Schwartz, F. W. (Hg.) (2011): Barmer GEK-Arztreport 2011. St. Augustin: Asgard-Verl.

Jacobs, Klaus (2010): Solidarität geht über Ländergrenzen. In: Gesundheit und Gesellschaft 13 (11), S. 14-15.
Medpac (2003): Variation and Innovation in Medicare. Report to the Congress. Medicare Payment Advisory Commission. Washington DC. National Health Service (2010): The NHS Atlas of Variation in Healthcare. Reducing unwarranted variation to increase value and improve quality.

Newhouse, Joseph P.; Garber, Alan M.; Graham, Robin P.; McCoy, Margaret A.; Mancher, Michelle; Kibria, Ashna (2013): Variation in health care spending. Target decision making, not geography.

Reschovsky, James D.; Hadley, Jack; Saiontz-Martinez, Cynthia B.; Boukus, Ellyn R. (2011): Following the money: factors associated with the cost of treating high-cost Medicare beneficiaries. In: Health Serv Res 46 (4), S. 997-1021.

Sheiner, Louise (2013): Why the Geographic Variation in Health Care Spending Can't Tell Us Much about the Efficiency or Quality of our Health Care System. Federal Reserve Board. Washington, D.C. (Finance and Economics Discussion Series).

Song, Yunjie; Skinner, Jonathan; Bynum, Julie; Sutherland, Jason; Wennberg, John E.; Fisher, Elliott S. (2010): Regional variations in diagnostic practices. In: N. Engl. J. Med. 363 (1), S. $45-53$.

Sutherland, Jason M.; Fisher, Elliott S.; Skinner, Jonathan S. (2009): Getting past denial--the high cost of health care in the United States. In: N. Engl. J. Med. 361 (13), S. $1227-1230$.

Wennberg, J.; Gittelsohn, A. (1973): Small area variations in health care delivery. In: Science 182 (4117), S. 1102-1108.

Wennberg, John E. (2010): Tracking medicine. $A$ researcher's quest to understand health care. New York: Oxford University Press.

\section{Zuckerman, Stephen; Waidmann, Timothy;} Berenson, Robert; Hadley, Jack (2010): Clarifying sources of geographic differences in Medicare spending. In: N. Engl. J. Med. 363 (1), S. $54-62$.

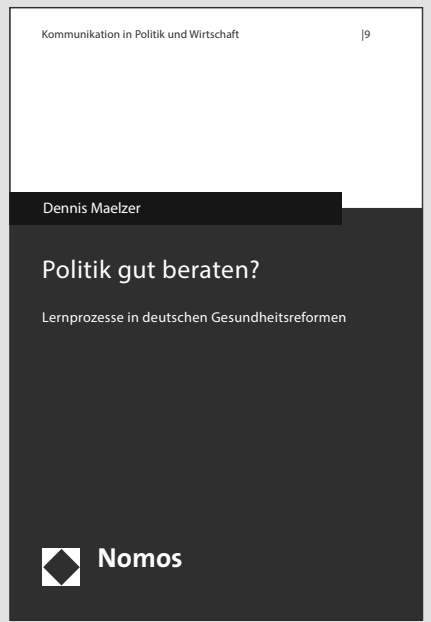

\section{Politik gut beraten?}

Lernprozesse in deutschen Gesundheitsreformen

Von Dennis Maelzer

2014, ca. 414 S., brosch., ca. 76,-€ ISBN 978-3-8487-1095-9

(Kommunikation in Politik und Wirtschaft, Bd. 9)

Erscheint ca. Februar 2014

Lernt Politik von der Wissenschaft? Das Veränderungspotential von Politikberatung beschäftigt Forscher, Berater und Politiker gleichermaßen. Der Autor analysiert, unter welchen Bedingungen PolicyLernprozesse angestoßen werden können. Sein Forschungsgebiet ist dabei die Gesundheitspolitik, die als pfadabhängig und konfliktträchtig zugleich gilt. 